\title{
Field-induced charge symmetry revealed by nuclear magnetic resonance in the topological insulator $\mathrm{Bi}_{2} \mathrm{Te}_{3}$
}

\author{
R. Guehne $\odot,{ }^{1, *}$ J. Haase, ${ }^{1}$ C. Shekhar, ${ }^{2}$ and C. Felser ${ }^{2}$ \\ ${ }^{1}$ Felix Bloch Institute for Solid State Physics, Leipzig University, Linnéstraße 5, 04103 Leipzig, Germany \\ ${ }^{2}$ Max Planck Institute for Chemical Physics of Solids, Nöthnitzer Straße 40, 01187 Dresden, Germany
}

(Received 31 August 2020; accepted 6 January 2021; published 19 February 2021)

\begin{abstract}
Nuclear magnetic resonance (NMR) was recently shown to measure the bulk band inversion of $\mathrm{Bi}_{2} \mathrm{Se}_{3}$ through changes in the ${ }^{209} \mathrm{Bi}$ nuclear quadrupole interaction, and the corresponding tensor of the local electric field gradient was found to follow, surprisingly, the direction of the external magnetic field if the sample is rotated. This manifests a hidden property of the charge carriers in the bulk of this topological insulator, which is explored here with another material, $\mathrm{Bi}_{2} \mathrm{Te}_{3}$. It is found that two electric field gradients appear to be present at ${ }^{209} \mathrm{Bi}$, one rests with the lattice, as usual, while a second follows the external field if it is rotated with respect to the crystal axes. These electronic degrees of freedom and their lifetime is believed to be responsible for an alternate quadrupolar relaxation that should lead to other special properties including the electronic specific heat.
\end{abstract}

DOI: 10.1103/PhysRevResearch.3.L012018

Gapless surface states of $\mathrm{Bi}_{2} \mathrm{Se}_{3}$-type three-dimensional topological insulators are of great interest from fundamental as well as applied physics' perspective [1], and they have been studied extensively with surface sensitive methods [2-4]. A spin-orbit coupling (SOC) induced energy band inversion at the $\Gamma$ point in the Brillouin zone of these materials was anticipated to lead to these topologically protected states [5]. However, related bulk properties of these materials have hardly been investigated.

Nuclear magnetic resonance (NMR) as a local, bulk probe was used, in a few applications, to study magnetic properties, in particular with aim at the surface states by using nanopowders, to increase the surface area [6,7]. However, definite conclusions are difficult as the NMR of spin-orbit coupled systems is not well understood [8], and bulk properties are expected to be altered by very small grains, as well [9]. For example, a special Bloembergen-Rowland type electronic susceptibility was found to be present in these narrow gap materials as it leads to large, field independent NMR linewidths [10].

Very recently, some of us have shown that NMR can indeed detect the band inversion of itinerant carriers via its effect on the local charge symmetry that influences the nuclear levels through the electric quadrupole interaction [11], leading to the well known splitting of NMR lines for spin $I>1 / 2$ nuclei [12]. This quadrupole splitting is known to be very sensitive to the local charge symmetry represented by the electric field gradient (EFG) at the nuclear site; it even detects lattice

\footnotetext{
*r.guehne@ physik.uni-leipzig.de

Published by the American Physical Society under the terms of the Creative Commons Attribution 4.0 International license. Further distribution of this work must maintain attribution to the author(s) and the published article's title, journal citation, and DOI.
}

strain arising from impurities. It is thus not surprising that quadrupole splittings also reflect changes of the electronic wave function due to SOC. Furthermore, the experiments can be reliably compared to first-principles calculations.

Very unusual, however, and unfortunately not easily tractable with first-principles calculations, is the orientation dependence of the quadrupole splitting in $\mathrm{Bi}_{2} \mathrm{Se}_{3}$ [11], i.e., if one changes the orientation of the crystal with respect the external magnetic field $\left(B_{0}\right)$. In NMR it is always assumed that the EFG, a second-rank tensor, rests with the crystal axes system. For example, this leads to typical powder spectra when the grains are oriented randomly, or, in case of single crystals, to special orientation dependences of the NMR spectra, which are a fingerprint of the local crystal symmetry. Surprisingly, for $\mathrm{Bi}_{2} \mathrm{Se}_{3}$, this tensor appears to follow the external field to a large extent, as shown in Fig. 1.

In leading order of the nuclear Zeeman interaction, the electric quadrupole splitting is usually expressed in terms of an orientation dependent quadrupole frequency $\left(v_{\mathrm{Q}}\right)$ given by the orientation dependent influence of the second rank tensor of the local electric field gradient $\left(V_{\mathrm{XX}}, V_{\mathrm{YY}}, V_{\mathrm{ZZ}}\right)$. The local symmetry at the Bi nucleus requires that $V_{\mathrm{XX}}=V_{\mathrm{YY}}$ and only one tensor component enters the well-known expression $v_{Q}=3 e Q V_{\mathrm{ZZ}} /(4 I(2 I-1) h) \cdot\left(3 \cos ^{2} \beta-1\right)$, where $I$ is the nuclear spin and $e Q$ the nuclear electric quadrupole moment. The azimuthal angle $\beta$ spans between $V_{\mathrm{ZZ}}$ and the external magnetic field $B_{0}$.

For ${ }^{209} \mathrm{Bi}$ in $\mathrm{Bi}_{2} \mathrm{Se}_{3}(I=9 / 2), V_{\mathrm{ZZ}}$ is strongly influenced by the band inversion and depends on the carrier concentration [11]. However, there is hardly any orientational dependence, i.e., $V_{\mathrm{ZZ}}$ remains nearly aligned with $B_{0}$, cf. Fig. 1(c). Interestingly, this phenomenon is most prominent for higher carrier concentrations, $n \geqslant 10^{19} \mathrm{~cm}^{-3}$ [11], so that one has to associate it with the doped electrons. It was argued that the system's massive SOC $\left(g_{\text {eff }} \approx 30\right.$ [14]) allows the external magnetic field to deform partially filled $\mathrm{Bi}$ orbitals. 


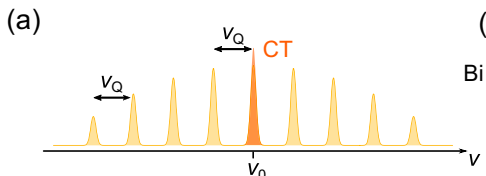

(c)

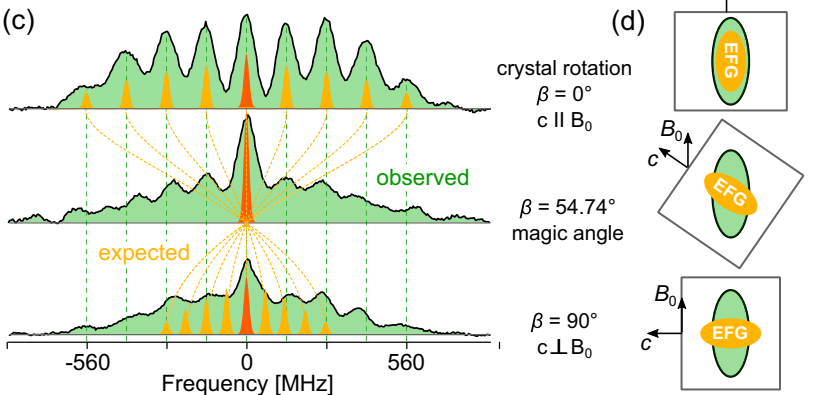

FIG. 1. ${ }^{209} \mathrm{Bi} \mathrm{NMR}$ of single crystalline $\mathrm{Bi}_{2} \mathrm{Se}_{3}$ at $11.74 \mathrm{~T}$ and $300 \mathrm{~K}$ : (a) expected quadrupole splitting, $v_{Q}$, in the high magnetic field $\left(B_{0}\right)$ case. The tensor of the electric field gradient $(E F G)$ at the $\mathrm{Bi}$ nucleus and its orientation with respect to the external field determine $v_{\mathrm{Q}}$. (b) the crystal symmetry at the Bi site and the band structure (band topology following [13]) affect $v_{\mathrm{Q}}$ through local changes in the wave function or carrier concentration [11]. (c) comparison with measurements (green spectra) are in quantitative agreement with DFT calculations [11], however, the expected orientation dependence of $\left(3 \cos ^{2} \beta-1\right)$ from this second rank tensor (orange spectra) is lacking, on the contrary, an orientation independent splitting (green spectra and lines) suggests that the EFG rotates with $B_{0}$, rather than being tied to the crystal, as depicted in panel (d).

The resulting EFG is then set by $B_{0}$, in stark contrast to an ordinary EFG that is pinned to the crystal axes. In other words, the effect involves rotational degrees of freedom of strongly spin-orbit coupled conduction electrons, with their orbital angular momenta being partially unquenched due to $B_{0}$ [15]. Earlier ${ }^{209} \mathrm{Bi}$ NMR studies of $\mathrm{Bi}_{2} \mathrm{Se}_{3}$ [16-19] are in agreement with the above-mentioned special results, but the short nuclear spin relaxation times for $\mathrm{Bi}$ apparently hindered a clear identification of this effect, before Ref. [18].

A magnetic field affecting the EFG is not uncommon. Field induced charge density waves in superconducting cuprates are an example [20,21]. In certain paramagnetic systems with a cubic local environment, exotic magnetic correlation patterns can also give rise to an EFG [22]. The ordinary first-order quadrupole interaction can show a magnetic field dependence, as well, if electronic wave functions are deformed and thus an EFG is induced even in closed shell systems such as noble gases [23]. Field induced charge rotation based on spin-orbit interaction has also been reported for impurity nuclei in ferromagnetic materials $[24,25]$. The effect as observed in $\mathrm{Bi}_{2} \mathrm{Se}_{3}$ is special, though, because it is related to rotational degrees of freedom of nonlocalized conduction electrons.

In order to shed more light on this unusual behavior, we decided to investigate $\mathrm{Bi}_{2} \mathrm{Te}_{3}$, the closest relative of $\mathrm{Bi}_{2} \mathrm{Se}_{3}$, with ${ }^{209} \mathrm{Bi}$ NMR. We find the quadrupole splitting to be an order of magnitude larger compared to that in $\mathrm{Bi}_{2} \mathrm{Se}_{3}$, and the orientation dependent splitting appears closer to what is expected from ordinary behavior. However, near the magic angle $\left(\cos ^{2} \beta=1 / 3\right)$, where the usual quadrupole splitting disappears, it becomes apparent that a quadrupolar split spectrum

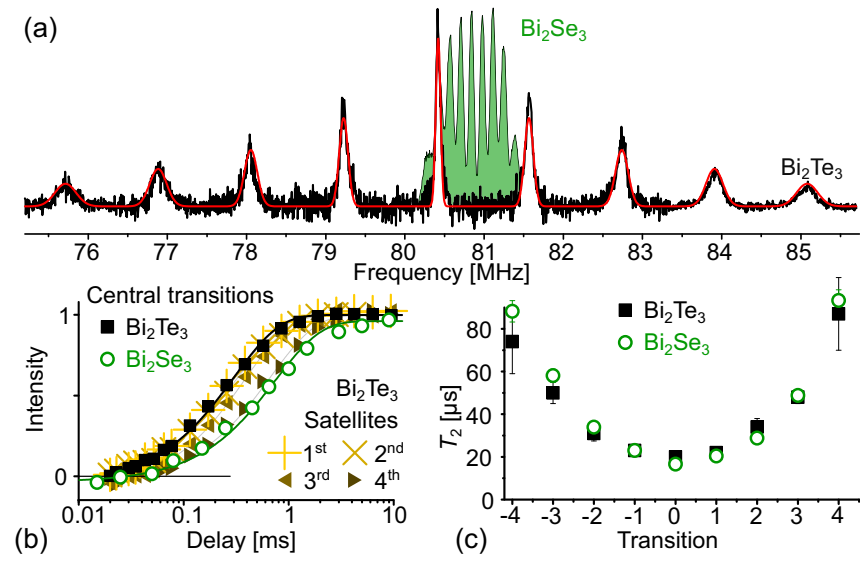

FIG. 2. (a) quadrupole spectrum of ${ }^{209} \mathrm{Bi}$ in $\mathrm{Bi}_{2} \mathrm{Te}_{3}$ (black) obtained for $c \| B_{0}$ at $11.74 \mathrm{~T}$ (selective spin echoes with $\pi / 2$-pulse width of $3 \mu \mathrm{s}$ ), including a fit (red line, see text for details). Also shown is a typical ${ }^{209} \mathrm{Bi}$ spectrum of single crystalline $\mathrm{Bi}_{2} \mathrm{Se}_{3}(\mathrm{~S} 3$ in [11]). (b) saturation recovery of the $\mathrm{Bi}$ central transitions in $\mathrm{Bi}_{2} \mathrm{Te}_{3}$ (squares) and $\mathrm{Bi}_{2} \mathrm{Se}_{3}$ (circles), respectively, next to the satellite recoveries in $\mathrm{Bi}_{2} \mathrm{Te}_{3}$ (triangles). (c) spin-echo decay times, $T_{2}$, for each of the $2 I=9 \mathrm{Bi}$ transitions in $\mathrm{Bi}_{2} \mathrm{Te}_{3}$ and $\mathrm{Bi}_{2} \mathrm{Se}_{3}$.

remains, similar to what was observed in $\mathrm{Bi}_{2} \mathrm{Se}_{3}$, manifesting a clear discrepancy with the expected behavior. In fact, a closer look at the total angular dependence shows this phenomenon also at other angles. Again, it appears that the electronic degrees of freedom change with the orientation of the external field, yielding a nonvanishing splitting even at the magic angle. In contrast to $\mathrm{Bi}_{2} \mathrm{Se}_{3}$, however, the quadrupole interaction in $\mathrm{Bi}_{2} \mathrm{Te}_{3}$ appears to have two EFGs, a large lattice component that rotates together with the sample, and a second component that follows the field. Hence, the ${ }^{209} \mathrm{Bi}$ quadrupole interaction in $\mathrm{Bi}_{2} \mathrm{Te}_{3}$ provides another perspective on a very unusual effect related to so far unknown electronic properties of conduction electrons in topological insulators.

$\mathrm{Bi}_{2} \mathrm{Te}_{3}$ used for the investigations here was grown by the self-flux method. A stoichiometry ratio of Bi (purity: 99.999\%) and Te (purity: $99.999 \%$ ) are sealed in a dry quartz ampoule with vacuum of $10^{-5}$ mbar. Except for the cooling rate of $2 \mathrm{~K} / \mathrm{h}$ from $800-500^{\circ}$ for the crystal growth, the other heating profile is the same as in Ref. [26]. A $c$-axis-oriented plateletlike crystal of several $\mathrm{mm}$ in dimension was mechanically separated from the ingot.

All experiments were carried out at room temperature with commercial NMR consoles using a home-built probe that fits standard 2.35 T, 7.05 T, 11.74 T, and 17.6 T magnets. We employed spin echoes $(\pi / 2-\tau-\pi)$ to measure individual transitions, their decay $\left(T_{2}\right)$, as well as their recovery after saturation $\left(T_{1}\right)$, besides, solid echoes $(\pi / 2-\tau-\pi / 2)$ to excite the whole set of transitions simultaneously where possible.

We start with ${ }^{209} \mathrm{Bi}$ NMR spectra of the $\mathrm{Bi}_{2} \mathrm{Te}_{3}$ single crystal with the magnetic field $B_{0}$ along the crystal $c$ axis ( $c \| B_{0}$ ), where $\beta=0$, cf. Fig. 2(a).

We find the expected $2 I=9$ resonances, and a simple fit of Gaussian lines to the total spectrum is in very good agreement with the expected pattern for a magnetic (the same for all transitions) and quadrupolar (contribution changes with transitions and is absent for the central line) 
broadening; we find $v_{Q}=1.171(2) \mathrm{MHz}$, a central transition linewidth of $80(2) \mathrm{kHz}$, and a relative quadrupole broadening of $7 \%$, i.e., about $80 \mathrm{kHz}$ for the first pair of satellites. For comparison, we show the spectrum for $\mathrm{Bi}_{2} \mathrm{Se}_{3}$, as well (sample $\mathrm{S} 3$ in Ref. [11]). The quadrupole splitting frequency is $0.14 \mathrm{MHz}$ with a central transition width of $77(4) \mathrm{kHz}$ and a relative quadrupolar broadening of up to $9 \%$, i.e., $\sim 13 \mathrm{kHz}$ for the first satellites [11]. Thus, while the quadrupole coupling and its distribution are nearly a factor of 10 larger in $\mathrm{Bi}_{2} \mathrm{Te}_{3}$, the magnetic (central) widths are similar. This NMR line broadening agrees with theoretical linewidths of an enhanced nuclear dipole coupling arising from the Bleombergen-Rowland electronic spin susceptibility $[10,27]$.

Comparison of the relaxation in $\mathrm{Bi}_{2} \mathrm{Te}_{3}$ and $\mathrm{Bi}_{2} \mathrm{Se}_{3}$ reveals similarities, as well. Selective recoveries of the central transitions are shown in Fig. 2(b), with apparent recovery times of 303(5) $\mu$ s for $\mathrm{Bi}_{2} \mathrm{Te}_{3}$ and 750(50) $\mu$ s for $\mathrm{Bi}_{2} \mathrm{Se}_{3}$. Since individual transitions are well separated in the case of $\mathrm{Bi}_{2} \mathrm{Te}_{3}$, we measured their recovery, as well (brown symbols). The recovery slows down with increasing satellite order, from $311(5) \mu \mathrm{s}\left(1^{\text {st }}\right.$ satellite $)$ over $337(5) \mu \mathrm{s}$ and $382(5) \mu \mathrm{s}\left(2^{\text {nd }}\right.$ and $3^{\text {rd }}$, respectively) to $672(10) \mu \mathrm{s}\left(4^{\text {th }}\right)$. Comparison with a purely magnetic relaxation $[17,28]$ based on the recovery of the central transition reveals discrepancies, especially for the outer satellites, indicating contributions from quadrupolar relaxation $[29,30]$.

Selective spin-spin relaxation experiments, Fig. 2(c), yield the same $T_{2}$ values within error for both samples, with a characteristic increase in $T_{2}$ with growing satellite order. The central transition $T_{2}$ values are further in good correlation with their width in frequency domain, and thus with the aforementioned model calculations based on the special Bloembergen-Rowland type of indirect nuclear dipole coupling.

We now turn to orientation dependent spectra shown in Fig. 3(b). The gray solid lines represent the expected resonance frequencies as a function of $\beta$, as obtained from exact diagonalization of the total Hamiltonian with $v_{\mathrm{Q}}=$ $1.171 \mathrm{MHz}$ for an axially symmetric field gradient, at a resonance frequency of $80.4 \mathrm{MHz}\left(B_{0}=11.74 \mathrm{~T}\right)$. While the central transition follows the predicted frequency dependence on angle [Fig. 4(a)], the satellite resonances do not. In particular, near the magic angle the total satellite spectral weight is distributed over a very large spectral range, while some asymmetry of the spectrum is expected from higher order effects. The broad spectral range and its asymmetry follow from broadband solid echo spectra as well as from frequency stepped, selective excitation. A more detailed orientation dependence near the magic angle is shown in panel $b$, with the expected (yellow area) and the measured (gray areas) spectral intensity (green line is the expectation for the central transition). With the magnetic field perpendicular to the $c$ axis the spectrum approaches what is expected from $c \| B_{0}$. The question arises how one can understand the disagreement, in particular near the magic angle.

Frequency stepped spin-echo spectra near the magic angle show, cf. Fig. 4(b), that the spectrum is independent of the field, at least for $B_{0} \geqslant 2 \mathrm{~T}$. Hence, it cannot stem from a distribution of shifts $\left(\propto B_{0}\right)$, or from an ordinary variation

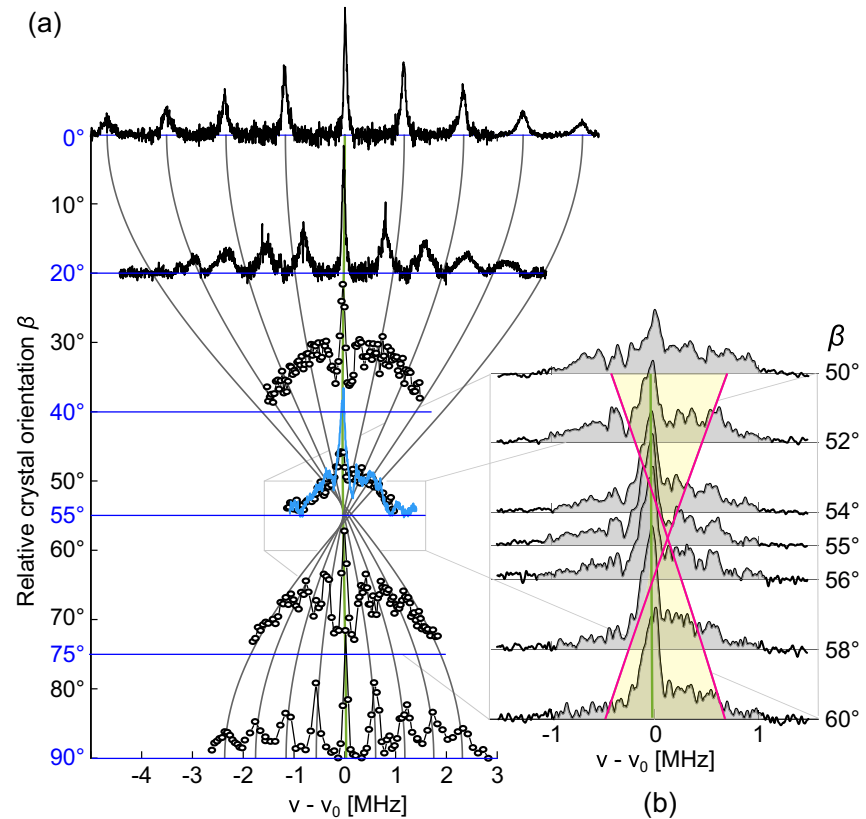

FIG. 3. Orientation dependent ${ }^{209} \mathrm{Bi}$ NMR spectra of $\mathrm{Bi}_{2} \mathrm{Te}_{3}$ (black spectra represent individually measured transitions, black circles frequency swept spectra, and the blue spectrum was obtained from a solid echo experiment). Main panel (a) the top trace for $c \| B_{0}$ $\left(\beta=0^{\circ}\right)$ is connected with what is expected from the quadrupole interaction by gray lines with the bottom trace $c \perp B_{0}\left(\beta=90^{\circ}\right)$. (b) shows a magnified window of solid echo experiments between $\beta=50^{\circ}$ and $60^{\circ}$. The two pink lines give the traces of the two outermost satellites, i.e., spectral density is only expected in the yellow area. In contrast, the measured spectra cover a much larger range of about $\pm 1 \mathrm{MHz}$ (gray filling).
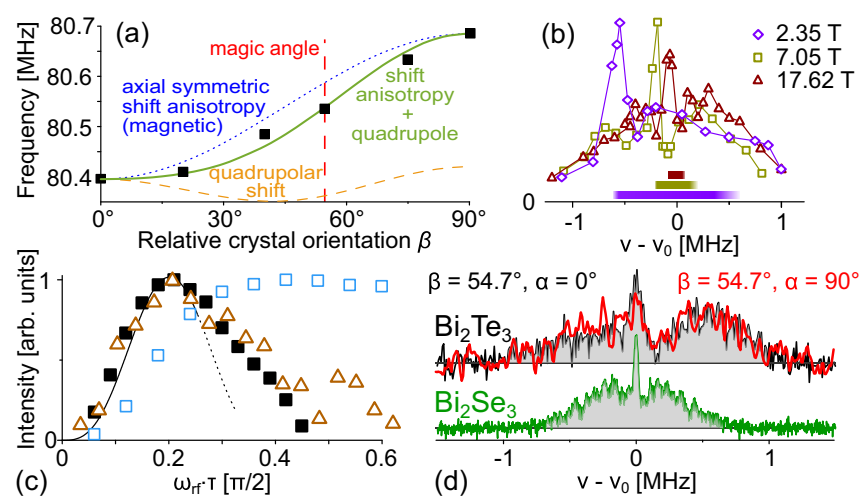

FIG. 4. (a) the resonance frequency of the central transition as a function of $\beta$ reflects an axial symmetric Knight shift anisotropy plus higher order quadrupole interaction (solid line). (b) frequency swept spin echoes spectra at $B_{0}=2.35 \mathrm{~T}$ (diamonds), $7.05 \mathrm{~T}$ (squares), and $17.6 \mathrm{~T}$ (triangles) for the magic angle. The spectra are shifted with respect to the center of gravity of their expected second-order quadrupole patterns, represented by the colored bars. (c) selective spin-echo nutation for the magic angle $+240 \mathrm{kHz}$ off center (triangles) in agreement with the central transition nutation for $c \| B_{0}$ (solid squares, line) confirms a quadrupolar origin of the broad magic angle spectrum. Consequently, broad band solid echo nutation (open squares) behaves differently, with a much slower nutation frequency. (d) magic angle broad band solid echo spectra for $\tau=32 \mu \mathrm{s}$ of $\mathrm{Bi}_{2} \mathrm{Te}_{3}\left(\alpha=0^{\circ}\right.$ and $\left.90^{\circ}\right)$ and $\mathrm{Bi}_{2} \mathrm{Se}_{3}$. 
of the quadrupole interaction $\left(\propto B_{0}^{-1}\right.$ for the second-order splitting at the magic angle). Selective nutation [Fig. 4(c)] reveals that the signal intensity belongs to single transitions, so it must be given by quadrupolar satellites that extend over a range of about $1 \mathrm{MHz}$. Note that the total spectral intensity remains constant as a function of $\beta$, thus, the observed effects concern the bulk signal. This means, the spectral intensity near the magic angle cannot be accounted for by sample inhomogeneity.

Obviously, the description above points immediately to a second quadrupolar broadening mechanism (of about $500 \mathrm{kHz}$ ) as it produces the desired broadening, i.e., the splitting up of the satellite transitions without affecting the central transition and keeping the asymmetry set by the center of gravity of the second-order satellite spectrum [colored bars in Fig. 4(b)]. However, this additional broadening must be rather independent from the main broadening mechanism and, given the lack of a special line shape, is likely to carry an additional inhomogeneity. Since the orientation dependence of the spectra is not very good at other angles, it is likely that the second term is already present far away from the magic angle, with a possible orientation dependent magnitude. For example, we find a much increased quadrupolar broadening even for $c \perp B_{0}$. In contrast, the rather narrow lines obtained for $c \| B_{0}$ may indicate that this orientation is exceptional because of the symmetry given by the similar coordination of the six Se neighbors with respect to $B_{0}$.

Note that symmetry in the plane is conserved as a rotation about the crystal $c$ axis $\left(\alpha=0^{\circ}, 90^{\circ}\right)$ inside the NMR coil does not change the spectrum, cf. Fig. 4(d), as expected.

Given the similarity of a nonvanishing magic angle quadrupole splitting in both materials, $\mathrm{Bi}_{2} \mathrm{Te}_{3}$ and $\mathrm{Bi}_{2} \mathrm{Se}_{3}$, cf. Fig. 4(d), and their close electronic relationship, i.e., very large effective $g$ factors of conduction electrons [14,31], we argue that quadrupole coupling that follows the field arises from the effect of the magnetic field on special electronic states, while the much stronger (normal) Bi quadrupole interaction in $\mathrm{Bi}_{2} \mathrm{Te}_{3}$ is related to a large $\mathrm{EFG}$ component from the ionic lattice. Note that for $\mathrm{Bi}_{2} \mathrm{Se}_{3}$ the effect of a nontrivial, orientation independent quadrupole splitting dominates the observation, with a negligible lattice EFG ( $\left.v_{Q}^{\text {latt }} \leqslant 10 \mathrm{kHz}\right)$. In $\mathrm{Bi}_{2} \mathrm{Te}_{3}$, the opposite situation holds, with a dominating lattice EFG yielding a first-order orientation dependence (with small second-order corrections), which gives way to a nontrivial EFG component from special electronic states (precessing about $B_{0}$ ) dominating the NMR near the magic angle.

In the last part of the discussion, we reconsider spin-lattice relaxation in the context of a special EFG arising from these electrons final lifetime of precession, as it must result in fluctuations of the EFG and thus nuclear relaxation. Results on the recovery of individually saturated $\mathrm{Bi}$ transitions in $\mathrm{Bi}_{2} \mathrm{Te}_{3}$ for $c \| B_{0}$ indicate a quadrupolar spin-lattice relaxation. The estimated recovery times of the central transitions in $\mathrm{Bi}_{2} \mathrm{Te}_{3}$ and $\mathrm{Bi}_{2} \mathrm{Se}_{3}$, however, are in clear disagreement with the measured quadrupole splittings for $c \| B_{0}$, because quadrupolar relaxation is expected to be proportional to $v_{Q}^{2}$. One may thus invoke contributions from magnetic relaxation. Though, when comparing the magic angle spectra for $\mathrm{Bi}_{2} \mathrm{Te}_{3}$ and $\mathrm{Bi}_{2} \mathrm{Se}_{3}$ in Fig. 4(d), both patterns are rather similar, the one obtained from $\mathrm{Bi}_{2} \mathrm{Te}_{3}$ is only slightly larger than the one from $\mathrm{Bi}_{2} \mathrm{Se}_{3}$. Hence, an alternative scenario to account for $\mathrm{Bi}$ spin-lattice relaxation in $\mathrm{Bi}_{2} \mathrm{Te}_{3}$ and $\mathrm{Bi}_{2} \mathrm{Se}_{3}$ could be related to the nontrivial EFG component that may be related to a special electronic thermal reservoir.

In summary, ${ }^{209} \mathrm{Bi} \mathrm{NMR}$ of $\mathrm{Bi}_{2} \mathrm{Te}_{3}$ reveals a nontrivial quadrupole interaction in the sense that the local charge distribution is altered by an external magnetic field. This bears strong similarities to what was observed in $\mathrm{Bi}_{2} \mathrm{Se}_{3}$, an electronically similar material. This behavior points to low energy electronic degrees of freedom in $\mathrm{Bi}_{2} \mathrm{Te}_{3}$ and $\mathrm{Bi}_{2} \mathrm{Se}_{3}$. Not only is this behavior crucial for the understanding and analysis of NMR data in such materials, it also raises the question of the role of these excitations for other material properties, for example, their so far unconsidered potential to contribute to the excellent thermoelectric performance [32].

R.G. and J.H. thank O. Sushkov, I. Garate, G. Williams, S. Chong, A. Pöppl, N. Georgieva, J. Nachtigal, A. Isaeva, and O. Oeckler, as well as the AMR group from the Felix Bloch Institute, Leipzig. We acknowledge the financial support by the Deutsche Forschungsgemeinschaft, Project No. 442459148, and by Leipzig University. The samples were provided for this study under the ERC Advanced Grant No. 742068 TOPMAT. We acknowledge support from Leipzig University for Open Access Publishing.
[1] Y. Ando, Topological insulator materials, J. Phys. Soc. Jpn. 82, 102001 (2013).

[2] Y. Xia, D. Qian, D. Hsieh, L. Wray, A. Pal, H. Lin, A. Bansil, D. Grauer, Y. S. Hor, R. J. Cava, and M. Z. Hasan, Observation of a large-gap topological-insulator class with a single Dirac cone on the surface, Nat. Phys. 5, 398 (2009).

[3] T. Zhang, P. Cheng, X. Chen, J. F. Jia, X. Ma, Ke He, L. Wang, H. Zhang, Xi Dai, Z. Fang, X. Xie, and Q. K. Xue, Experimental Demonstration of Topological Surface States Protected by Time-Reversal Symmetry, Phys. Rev. Lett. 103, 266803 (2009).
[4] F. Zhang, C. L. Kane, and E. J. Mele, Surface states of topological insulators, Phys. Rev. B 86, 081303(R) (2012).

[5] H. Zhang, C.-X. Liu, X.-L. Qi, Xi Dai, Z. Fang, and S.-C. Zhang, Topological insulators in $\mathrm{Bi}_{2} \mathrm{Se}_{3}, \mathrm{Bi}_{2} \mathrm{Te}_{3}$ and $\mathrm{Sb}_{2} \mathrm{Te}_{3}$ with a single Dirac cone on the surface, Nat. Phys. 5, 438 (2009).

[6] D. Koumoulis, T. C. Chasapis, R. E. Taylor, M. P. Lake, D. King, N. N. Jarenwattananon, G. A. Fiete, M. G. Kanatzidis, and Louis-S. Bouchard, NMR Probe of Metallic States in Nanoscale Topological Insulators, Phys. Rev. Lett. 110, 026602 (2013). 
[7] W. Papawassiliou, A. Jaworski, A. J. Pell, J. H. Jang, Y. Kim, S.-C. Lee, H. J. Kim, Y. Alwahedi, S. Alhassan, A. Subrati, M. Fardis, M. Karagianni, N. Panopoulos, J. Dolinšek, and G. Papavassiliou, Resolving Dirac electrons with broadband highresolution NMR, Nat. Commun. 11, 1 (2020).

[8] S. Boutin, J. Ramírez-Ruiz, and I. Garate, Tight-binding theory of NMR shifts in topological insulators $\mathrm{Bi}_{2} \mathrm{Se}_{3}$ and $\mathrm{Bi}_{2} \mathrm{Te}_{3}$, Phys. Rev. B 94, 115204 (2016).

[9] L. Gioia, M. G. Christie, U. Zülicke, M. Governale, and A. J. Sneyd, Spherical topological insulator nanoparticles: Quantum size effects and optical transitions, Phys. Rev. B 100, 205417 (2019).

[10] N. M. Georgieva, D. Rybicki, R. Guehne, G. V. M. Williams, S. V. Chong, K. Kadowaki, I. Garate, and J. Haase, ${ }^{77}$ Se nuclear magnetic resonance of topological insulator $\mathrm{Bi}_{2} \mathrm{Se}_{3}$, Phys. Rev. B 93, 195120 (2016).

[11] R. Guehne, V. Chlan, G. V. M. Williams, S. V. Chong, K. Kadowaki, A. Pöppl, and J. Haase, Unusual ${ }^{209}$ Bi NMR quadrupole effects in topological insulator $\mathrm{Bi}_{2} \mathrm{Se}_{3}$, J. Magn. Reson. 302, 34 (2019).

[12] Charles P. Slichter, Principles of Magnetic Resonance, third enlarged ed. (Springer, Berlin, 1990).

[13] C.-X. Liu, X.-L. Qi, H. J. Zhang, Xi Dai, Z. Fang, and S.C. Zhang, Model hamiltonian for topological insulators, Phys. Rev. B 82, 045122 (2010).

[14] H. Köhler and E. Wöchner, The g-factor of the conduction electrons in $\mathrm{Bi}_{2} \mathrm{Se}_{3}$, Phys. Status Solidi B 67, 665 (1975).

[15] O. Sushkov (private communication).

[16] B.-L. Young, Z.-Yo Lai, Z. Xu, A. Yang, G. D. Gu, Z.-H. Pan, T. Valla, G. J. Shu, R. Sankar, and F. C. Chou, Probing the bulk electronic states of $\mathrm{Bi}_{2} \mathrm{Se}_{3}$ using nuclear magnetic resonance, Phys. Rev. B 86, 075137 (2012).

[17] D. M. Nisson, A. P. Dioguardi, P. Klavins, C. H. Lin, K. Shirer, A. C. Shockley, J. Crocker, and N. J. Curro, Nuclear magnetic resonance as a probe of electronic states of $\mathrm{Bi}_{2} \mathrm{Se}_{3}$, Phys. Rev. B 87, 195202 (2013)

[18] D. M. Nisson, A. P. Dioguardi, X. Peng, D. Yu, and N. J. Curro, Anomalous nuclear magnetic resonance spectra in $\mathrm{Bi}_{2} \mathrm{Se}_{3}$ nanowires, Phys. Rev. B 90, 125121 (2014).

[19] S. Mukhopadhyay, S. Krämer, H. Mayaffre, H. F. Legg, M. Orlita, C. Berthier, M. Horvatić, G. Martinez, M. Potemski, B. A. Piot et al., Hyperfine coupling and spin polarization in the bulk of the topological insulator $\mathrm{Bi}_{2} \mathrm{Se}_{3}$, Phys. Rev. B 91, 081105(R) (2015).
[20] T. Wu, H. Mayaffre, S. Krämer, M. Horvatić, C. Berthier, W. N. Hardy, R. Liang, D. A. Bonn, and M.-H. Julien, Magnetic-field-induced charge-stripe order in the hightemperature superconductor $\mathrm{YBa}_{2} \mathrm{Cu}_{3} \mathrm{O}_{y}$, Nature 477, 191 (2011).

[21] S. Reichardt, M. Jurkutat, R. Guehne, J. Kohlrautz, A. Erb, and J. Haase, Bulk charge ordering in the $\mathrm{CuO}_{2}$ plane of the cuprate superconductor $\mathrm{YBa}_{2} \mathrm{Cu}_{3} \mathrm{O}_{6.9}$ by high-pressure NMR, Condens. Matter 3, 23 (2018).

[22] Y. Tokunaga, D. Aoki, Y. Homma, S. Kambe, H. Sakai, S. Ikeda, T. Fujimoto, R. E. Walstedt, H. Yasuoka, E. Yamamoto, A. Nakamura, and Y. Shiokawa, NMR Evidence for HigherOrder Multipole Order Parameters in $\mathrm{NpO}_{2}$, Phys. Rev. Lett. 97, 257601 (2006)

[23] J. Vaara and P. Pyykkö, Magnetic-field-induced quadrupole splitting in gaseous and liquid ${ }^{131} \mathrm{Xe}$ NMR: Quadratic and Quartic Field Dependence, Phys. Rev. Lett. 86, 3268 (2001).

[24] M. Aiga and J. Itoh, Nuclear electric quadrupole interaction of Ir nuclei in ferromagnetic dilute alloys of iron and nickel, J. Phys. Soc. Jpn. 37, 967 (1974).

[25] G. Seewald, E. Zech, and H. Haas, Spin-orbit induced noncubic charge distribution in cubic ferromagnets. II. Tight-binding analysis, Phys. Rev. B 66, 174402 (2002).

[26] C. E. ViolBarbosa, C. Shekhar, B. Yan, S. Ouardi, Eiji Ikenaga, G. H. Fecher, and C. Felser, Direct observation of band bending in the topological insulator $\mathrm{Bi}_{2} \mathrm{Se}_{3}$, Phys. Rev. B 88, 195128 (2013).

[27] J. Ramírez-Ruiz, C. Gauvin-Ndyae, and I. Garate (private communication).

[28] M. Takigawa, J. L. Smith, and W. L. Hults, Temperaturedependent anisotropy of $\mathrm{Cu}(2)$ nuclear-relaxation rate in $\mathrm{YBa}_{2} \mathrm{Cu}_{3} \mathrm{O}_{7}$ below $T_{c}$, Phys. Rev. B 44, 7764 (1991).

[29] J. Van Kranendonk, Theory of quadrupolar nuclear spin-lattice relaxation, Physica 20, 781 (1954).

[30] A. Suter, M. Mali, J. Roos, and D. Brinkmann, Mixed magnetic and quadrupolar relaxation in the presence of a dominant static Zeeman Hamiltonian, J. Phys.: Condens. Matter. 10, 5977 (1998).

[31] H. Köhler, Anisotropic g-factor of the conduction electrons in $\mathrm{Bi}_{2} \mathrm{Te}_{3}$, Phys. Status Solidi B 75, 127 (1976).

[32] C. Fu, Y. Sun, and C. Felser, Topological thermoelectrics, APL Mater. 8, 040913 (2020). 